\title{
Calibration of the ATLAS
Muon Chambers
}

Felix Rauscher

Ludwig-Maximilians-Universität München

on behalf of the ATLAS Muon Collaboration

$$
\text { 2009-10-07 }
$$




\section{Introduction}




\section{ATLAS Muon Spectrometer}

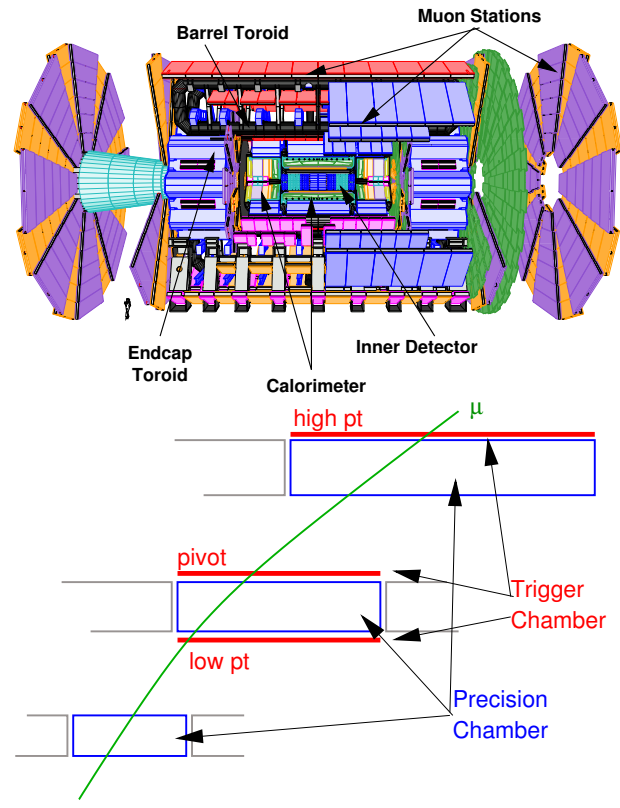

\section{Standalone Muon Spectrometry}

- $\Delta p_{T} / p_{T}<10 \%$ up to $1 \mathrm{TeV}$

- Coverage $|\eta|<2.7\left(|\theta|<86^{0}\right)$

\section{Layout}

- superconducting air coil toroid

- trigger chambers: RPC (barrel), TGC (end-cap)

- precision chambers: MDT, CSC (inner end-cap)

B-field in air reduces multiple

scattering, but high precision tracking $(50 \mu \mathrm{m})$ is required. 


\section{MDT - Drift Tube Chambers}

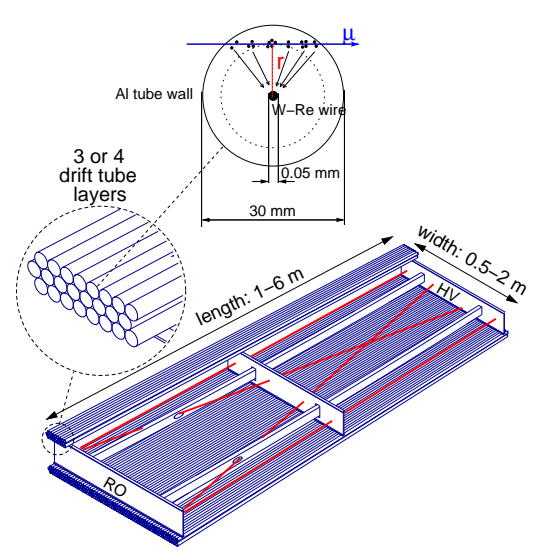

\section{Drift Tube}

- $\mathrm{Ar} / \mathrm{CO}_{2}(93 / 7)$ @ 3 bar absolute

- $\varnothing_{\text {Tube }}=30 \mathrm{~mm} \varnothing_{\text {Wire }}=50 \mu \mathrm{m}$

- $\mathrm{HV}=3080$

- Gas Gain: $210^{4}$

- Resolution: $80 \mu \mathrm{m}$ Requires rt-precision of $20 \mu \mathrm{m}$

\section{MDT-chamber}

- 2 multi-layers with 3 or 4 layers.

- surface $0.5-11 \mathrm{~m}^{2}$

- Monitoring of geometry, temperature, B-field. 


\section{Resolution Requirements}

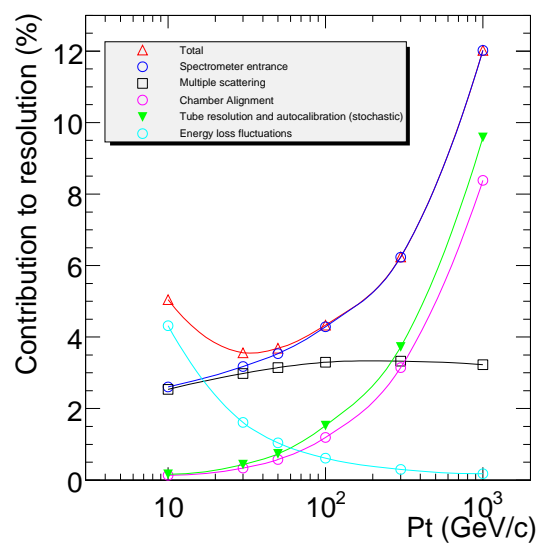

\section{First Collisions}

- $p_{T}<100 \mathrm{GeV}$

$\Rightarrow$ Resolution dominated by MS, and energy loss.

- required rt-precision: $O(100 \mu \mathrm{m})$

\section{Design Parameters}

- $p_{T}$ in TeV-range $\Rightarrow$ Resolution dominated by spacial resolution.

- required rt-precision: $O(20 \mu \mathrm{m})$ 


\section{$t_{0}-F i t$}

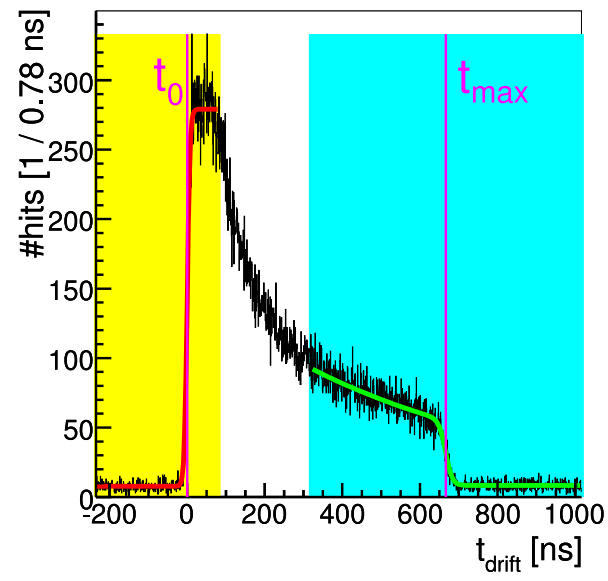

- Compensate for signal-propagation time in cables and electronics

- Required precision 0.5 ns.

Fit Fermi-like functions to leading and trailing edges of the drift time spectrum.

- Fit regions and start values are automatically determined.

- Leading edge position is the calibration.

- Slopes and length of spectrum are input to DQA. 


\section{rt-calibration}

Integration of drift-time spectrum

- Gives start rt with precision $O(100 \mu \mathrm{m})$

\section{Auto-calibration}

- Fit segments using input rt.

- deviation of $r_{\text {drift }}$ from $r_{\text {segment }}$ gives correction.

- iterate.

Resolution Determination

- Fit segment omitting one tube

- $\sigma($ residual $)=$ $\sigma\left(r_{\text {drift }}\right) \oplus \sigma\left(r_{\text {segment }}\right)$

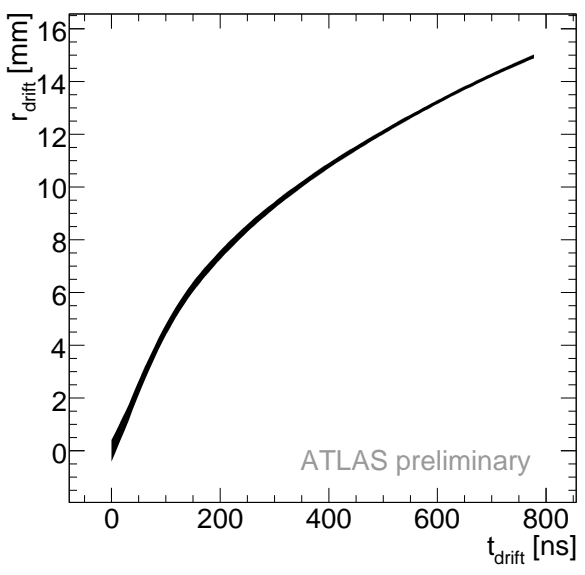

- iterate.

Required precision $20 \mu \mathrm{m}$ - to be done within $24 \mathrm{~h}$ after data-taking 


\section{Rt-Correction Functions}

One rt-relation is fitted by chamber. To compensate for effects, that are inhomogeneous insider one chamber, rt-correction functions are applied.

- wire-sag

- Barrel: Bend tubes, so that the wire is always in the middle.

- End-Cap: Apply rt-correction.

- temperature

- magnetic field 


\section{Calibration Stream}

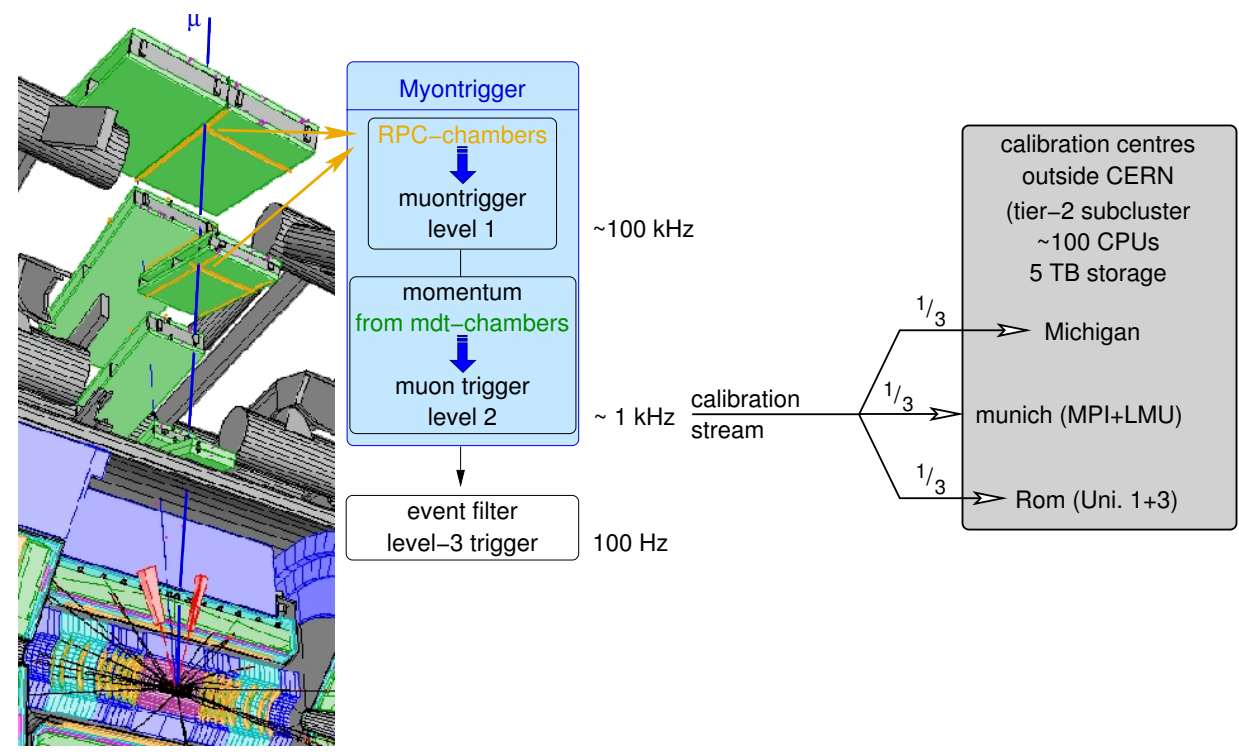




\section{Calibration During Commissioning with Cosmics}




\section{$t_{0}$-Performance}

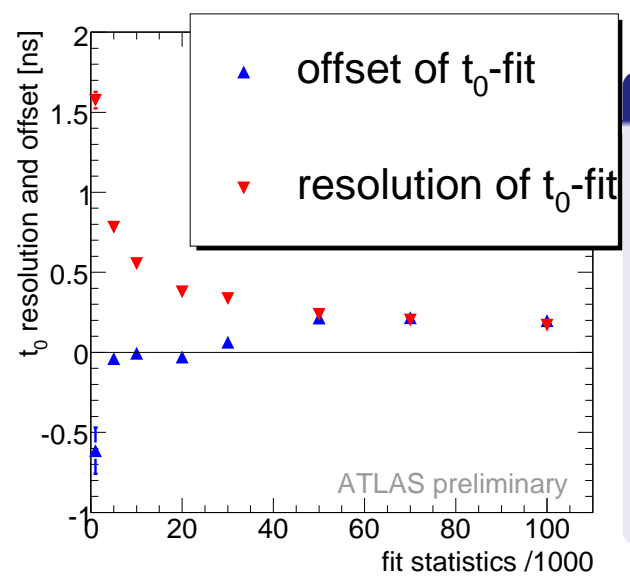

\section{Method}

- Fill a drift time spectrum with high statistics using cosmic data.

- Use this spectrum as input probability distribution, and create random drift times.

- Fill for a given fit statistics 20000 spectra.

- Analyse distribution of fitted t0.

- Required precision reached with 10000 hits per fit.

- Systematic deviation of t0 is negligible.

Per tube fit cannot be done with first data. Use cosmics and tof-corrections, or cosmics and per chamber t0. 


\section{rt-Performance}

\section{Residual Distribution}

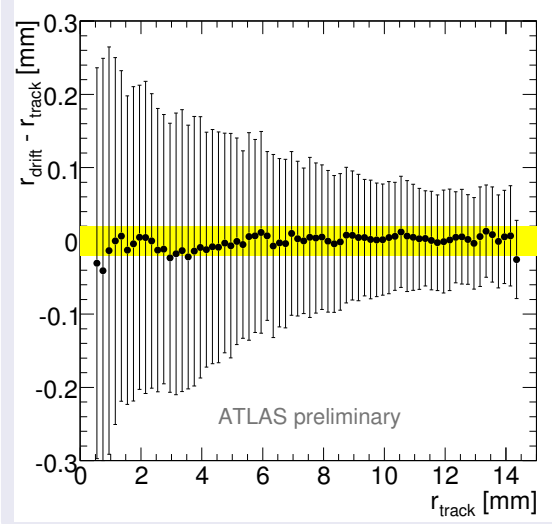

- Residual: $r_{\text {segment }}-r_{\text {drift }}$.

$\leftarrow$ Mean value and width of residual distribution vs $r$. Mean value shows systematic deviation.

- Precision of rt-distribution is $20 \mu \mathrm{m}$ 


\section{B-Field Correction}

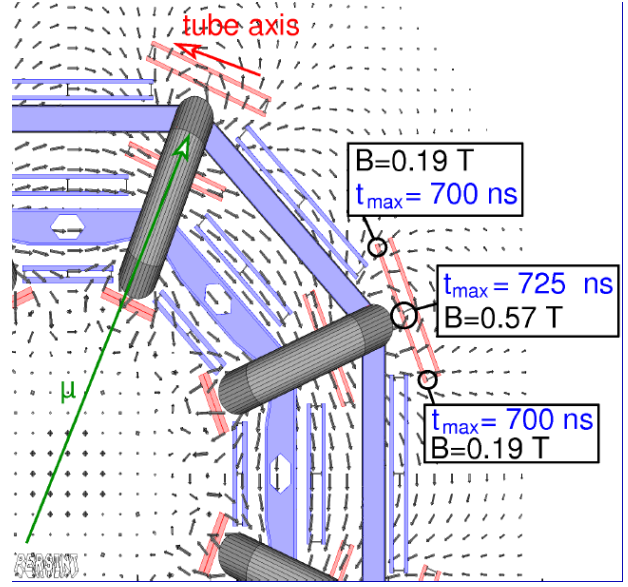

Necessity

- Correction to gasmonitor-rt for first collisions.

- B-field is not constant inside one chamber.

$\rightarrow 200 \mu \mathrm{m}$ rt-error for some chambers if not corrected

Strategy

- Do rt-calibration with B-field corrected drift times.

- Test: Look at middle chamber, to check systematic effect of b-field on rt-relation. 


\section{B-Field Correction}

Apply an rt-relation obtained from a run where the toroid was off to a run where the toroid was on:

\section{No B corrections}

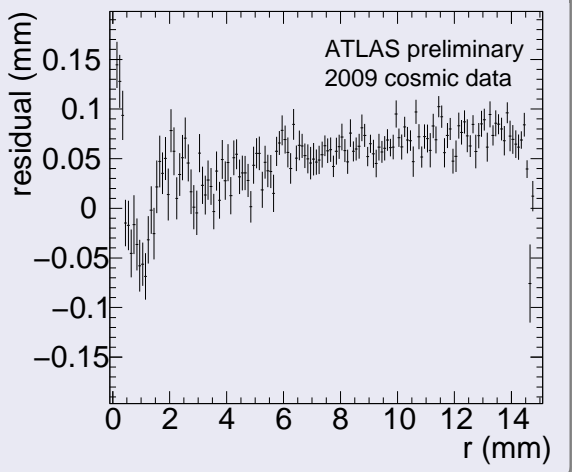

Rt-resolution of 20 to $50 \mu \mathrm{m}$.

\section{With B corrections}

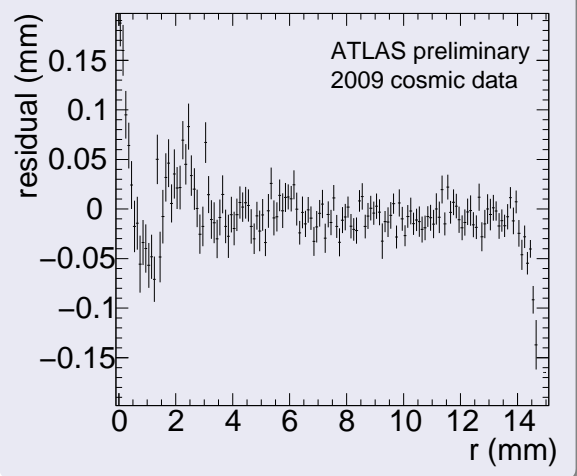




\section{Calibration of First Collisions}




\section{Strategy}

$10^{29} \mathrm{~cm}^{-2} \mathrm{~s}^{-1} @ 7 \mathrm{TeV} \approx 100$ Segment per Chamber in 2 days

- Use segment t0 in reconstruction.

- Use gas monitor rt and corrections.

$10^{30} \mathrm{~cm}^{-2} \mathrm{~s}^{-1} @ 7 \mathrm{TeV} \approx 1000$ Segment per Chamber in 2 days

- Use segment t0-fitting in calibration to determine a chamber-wise t0.

- Use gas monitor rt and corrections.

$10^{31} \mathrm{~cm}^{-2} \mathrm{~s}^{-1} @ 7 \mathrm{TeV} \approx 10000$ Segment per Chamber in 2 days

- Fit t0 for a longer period.

- Standard rt-calibration.

Rt precision will be at $100 \mu \mathrm{m}$ for $10^{30} \mathrm{~cm}^{-2} \mathrm{~s}^{-1}$ and below. Design rt-precision of $20 \mu \mathrm{m}$ is reached at $10^{31} \mathrm{~cm}^{-2} \mathrm{~s}^{-1}$ 


\section{Gas Monitor Chamber}
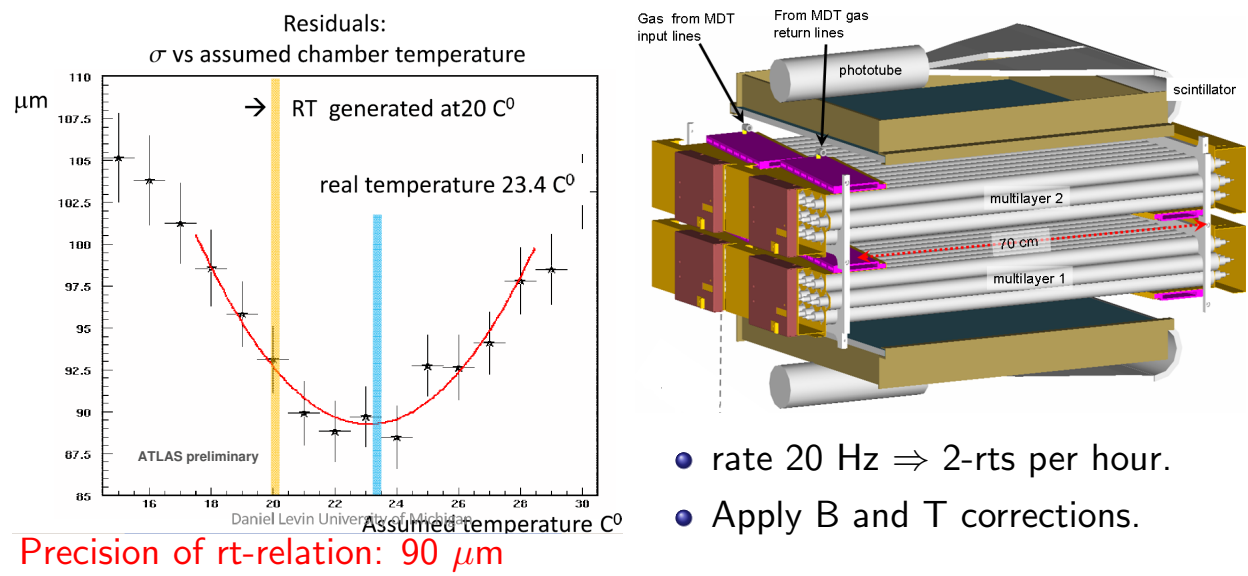

- rate $20 \mathrm{~Hz} \Rightarrow 2$-rts per hour.

- Apply B and T corrections. 


\section{Summary}

- Infrastructure and algorithms for the calibration of the drift tube chambers are in place and tested with cosmics.

- Precision of the calibration will be as foreseen at luminosities greater then $10^{31} \mathrm{~cm}^{-2} \mathrm{~s}^{-1}$ @ $7 \mathrm{TeV}$

- For first collisions, the rt precision will be at $100 \mu \mathrm{m}$. 